\title{
Study of the Spatiotemporal Behavior of LED-Pumped Ce:Nd:YAG Laser
}

\author{
Mostafa Tarkashvand $^{\mathrm{a}, \mathrm{b}}$, Amir Hossein Farahbod ${ }^{\mathrm{a}}$, and Seyed Ali Hashemizadeh $^{\mathrm{b}}$ \\ ${ }^{a}$ Department of plasma and nuclear fusion, Nuclear science technology research institute, \\ North Kargar, Tehran, Iran \\ ${ }^{b}$ Department of Physics, Payame Noor University, P.O. Box 19395-3697, Tehran, Iran
}

*Corresponding author E-mail: afarahbod@aeoi.org.ir

Regular paper: Received: Sep. 2, 2019, Revised: Dec. 6, 2019, Accepted: Aug. 3, 2020, Available Online: Aug. 4, 2020, DOI: 10.29252/ijop.14.1.75

\begin{abstract}
In this paper, the mode structure and time behavior of a LED-pumped Ce:Nd:YAG laser have been studied. Four blue LED bars with total 128 LEDs at $460 \mathrm{~nm}$ are utilized to pump a $3 \mathrm{~mm}$ diameter laser rod. Using a $\mathrm{Cr}^{4+}$ :YAG passive optical switch with 96\% initial transmission, and a low loss stable optical resonator and $0.7 \mathrm{~J}$ pumping energy, a single 17 micro-joules Q-switched laser pulse with 240 ns pulse-width and nearly TEM $_{00}$ mode profile was produced. By increasing the pumping energy $E_{p}$ up to $0.8 \mathrm{~J}$, the mode structure remained intact. Further increasing of $E_{p}$, the laser mode changed to TEM $_{10}$. Numerical calculations show that the central high gain area of the laser rod and saturation mechanism of the passive $Q$-switch behaves like as a soft aperture to enforce the laser resonator to oscillate on a low order transverse mode. For laser free-running, the TEM $_{00}$ mode has not been achieved and the optical resonator produced high order transverse mode patterns.
\end{abstract}

KeYwORDS: Ce:Nd:YAG laser, Cr:YAG passive Q-switch, LED-pumping, laser freerunning.

\section{I.INTRODUCTION}

Progress of light emitting diode (LED) technology during last decade is promising to make powerful, low cost, compact and highquality LED-pumped all solid-state lasers in the near future. Recently, several authors have described the LED-pumped Nd:YAG, Ce:Nd:YAG and Nd:YVO ${ }_{4}$ lasers with several hundred micro-joules to milli-joules level of laser energy [1]-[8]. Different LED spectrum from blue at $460 \mathrm{~nm}$ [2], to near infrared spectrum at 750 and $810 \mathrm{~nm} \mathrm{[3],} \mathrm{[7],} \mathrm{[9],} \mathrm{have}$ been used for the optical pumping of the solidstate active medium. Moreover, green and amber LEDs at 520 and $592 \mathrm{~nm}$, respectively [4], have been applied for the laser pumping. In addition, the optical pump efficiency up to better than 20 percent was reported [7].

Laser Q-switching technique by using passive optical switches is a reliable approach to obtain laser pulses of the order 1-100 ns [9]. The reports concerned with a passively Qswitched LED-pumped laser are given by Cho et al. [7] and Pichon et al. [8]. However, because of non-uniform pumping of the $\mathrm{Nd}$ :YAG active medium, the laser beam quality of Ref. [7] was very low (M-square factor about 40) and laser time behavior was not acceptable. Special pumping design of Ref. [8] by using Ce:YAG luminescent concentrators, and a very small dimensions $\left(1 \times 2.5 \times 14 \mathrm{~mm}^{3}\right)$ of $\mathrm{Nd}$ :YAG crystal, highly improved the beam quality and laser pulseshape. Recently, mode-quality and energy enhancement of a Q-switched LED-pumped at $810 \mathrm{~nm} \mathrm{Nd}$ :YAG laser has been reported [9].

The paper mainly presents the experimental observations and analysis of mode structure and time behavior of a LED-pumped Ce:Nd:YAG laser [5], [6]. The geometrical configuration of the LED sources and the optical resonator are similar to Refs. [4]-[6]. The high absorption of pumping radiations by 
the active medium, and the presence of passive Q-switch, results in a nearly Gaussian mode profile. The paper arranged as follows: section 2 describes the optical configuration of pump sources and resonator. The experimental findings are given in section 3. Comparison of the numerical calculations and experimental data appeared in section 4. Finally, the paper is concluded in section 5 .

Table 1. The calculated absorption efficiency of Ce:Nd:YAG and $N d: Y A G$ versus wavelength of LED peak emission.

\begin{tabular}{ccc}
\hline \hline$\lambda_{\mathrm{p}}(\mathrm{nm})$ & $\eta_{\mathrm{a}}(\%)$, Nd:YAG & $\eta_{\mathrm{a}}(\%)$, Ce: Nd:YAG \\
\hline \hline 460 & 3.2 & 61 \\
520 & 9.6 & 19.6 \\
592 & 16.3 & 24.5 \\
730 & 14.6 & 24.1
\end{tabular}

\section{EXPERIMENTAL SETUP}

The double-doped crystal Ce:Nd:YAG as a laser active medium has effective absorption bands near $460 \mathrm{~nm}$, which result from the efficient energy transfer among $\mathrm{Ce}^{3+}$ ions as sensitizer and $\mathrm{Nd}^{3+}$ active ions [10]. Therefore, we have used LED bars with significant power emission at $460 \mathrm{~nm}$ to pump optically a $2 a_{r}=3 \mathrm{~mm}$ diameter Ce:Nd:YAG laser rod with 0.1 and 1.0 atomic percent concentration of $\mathrm{Ce}^{3+}$, and $\mathrm{Nd}^{3+}$, respectively. Fig. 1 shows the normalized spectral power of the blue LED at $460 \mathrm{~nm}$ and the absorption coefficient of Ce:Nd:YAG that is calculated from the transmission curve of a Ce:Nd:YAG slab with thickness $\rho=3 \mathrm{~mm}$ [2].

The absorption efficiency $\eta_{a}$ for a slab of active medium with thickness $\rho$, absorption coefficient $\alpha(v)$ where $v$ is radiation frequency and the LED spectral power $E(v)$, is defined by Eq. (1) [4]. We have calculated the absorption efficiency of $\mathrm{Nd}: \mathrm{YAG}$ and Ce:Nd:YAG for some other LEDs with different peak emission wavelength $\lambda_{p}$ and spectral characteristics.

$$
\eta_{a}=\int_{0}^{\infty} E(v)\left(1-e^{-\alpha(v) \rho}\right) d v / \int_{0}^{\infty} E(v) d v
$$

The results are compared in Table 1 to evaluate the effect of $\mathrm{Ce}^{3+}$ on the pumping performance. It is seen that the pumping of Ce:Nd:YAG at $460 \mathrm{~nm}$ is drastically more effective than $\mathrm{Nd}$ :YAG pumping with green LED at $520 \mathrm{~nm}$. In addition, for other pumping wavelengths, the presence of $\mathrm{Ce}^{3+}$ has a remarkable effect on the absorption efficiency.

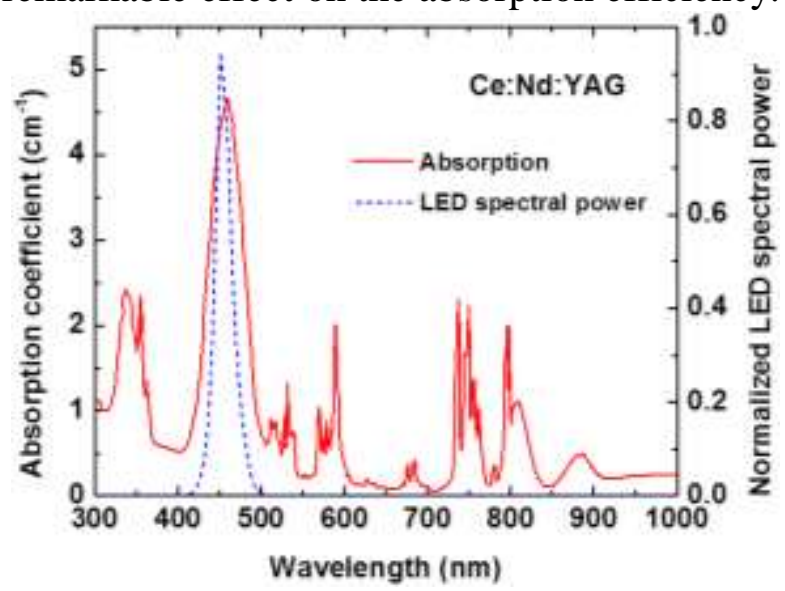

Fig. 1. Absorption spectrum of Ce:Nd:YAG crystal with 0.1 and 1.0 atomic percent concentration of $\mathrm{Ce}^{3+}$ and $\mathrm{Nd}^{3+}$, respectively and normalized spectral power of the blue LED with peak emission at 460 nm [2].

We have used four LED bars to side pump the $L_{a m}=60 \mathrm{~mm}$ length Ce:Nd:YAG active medium. Each LED bar is prepared from two segments of the blue section of RGB LED matrix of EPILEDS company type NE50WFGB-8C6B where carefully cut and electrically connected in series [4]. Each segment has 16 LEDs with minimum luminous flux $320 \mathrm{~lm}$ per segment. Therefore, we have used 128 LEDs to pump the active medium. The continuous forward current and voltage of the LED segments are $700 \mathrm{~mA}$ and $25 \mathrm{~V}$, respectively. The four LED bars are electrically in parallel and the total discharge current has been measured with a calibrated Rogowski current monitor.

The electrical pumping energy is given by $E_{p}=I_{L E D} V_{c h} t_{p}$, where $I_{L E D}, V_{c h}$, and $t_{p}$ are discharge current, charging voltage of the capacitor bank and current pulse duration, respectively. Discharge current with $t_{p}=318 \mu \mathrm{s}$ is at least one order of magnitude greater than the continuous forward current. 
The outer surface of laser rod is placed nearly in touch with the emitting surface of LEDs to overcome the low optical coupling efficiency results from 120 degrees angular radiation pattern of the light emitting diodes. No special effort is carried out for cooling the LED bars. Therefore, we have derived the LEDs in a very low repetition rate $0.1 \mathrm{~Hz}$, and discharge current limited to safe region to avoid overheating and damage of the LED chips.

The optical resonator is a low loss stable resonator [4-6]. Therefore, to achieve the laser oscillation and high resonator stability, the geometrical length of the resonator is chosen as low as possible to reduce the diffraction loss and resonator misalignment sensitivity. Output coupler (OC) is a flat mirror with $93 \%$ reflectivity at $1064 \mathrm{~nm}$, and back mirror (BM) is a concave total reflector with $R_{b m}=500 \mathrm{~mm}$ radius of curvature, Fig. 2 . The geometrical length of the resonator $L_{g}$ is 140 $\mathrm{mm}$. Hence, the geometrical parameter $g_{o c} g_{b m}=1-L_{g} / R_{o c}=0.72$ satisfies the resonator stability condition $0<g_{o c} g_{b m}<1$.

The passive Q-switch (PQS) is a $\mathrm{Cr}^{4+}: \mathrm{YAG}$ crystal with an initial transmission $T_{o}=0.96$, and its optical surfaces have anti-reflection coating at $1064 \mathrm{~nm}$.

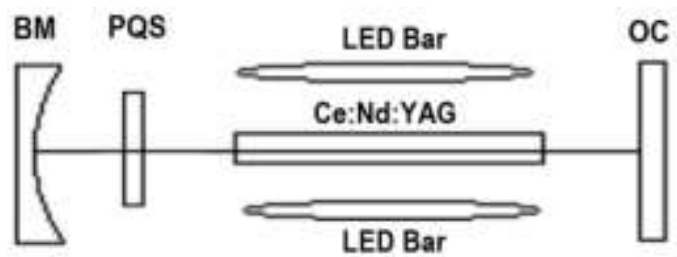

Fig. 2. Schematic of the Q-switched LED-pumped Ce:Nd:YAG laser oscillator.

\section{III.EXPERIMENTAL FINDINGS}

We have observed more than 450 micro-joules laser spiking for the highest safe discharge current of LEDs at $I_{L E D}=40 \mathrm{~A}$, that is equal to $E_{p}=1.6 \mathrm{~J}$ electrical pumping energy, Fig. 3. The electrical to optical (laser) conversion efficiency $\eta=E_{\text {laser }} / E_{p}$, has the highest value $3.5 \times 10^{-2} \%$ at $E_{p}=1.1 \mathrm{~J}$. The experimental findings show that with similar optical losses and equal pump geometry, the conversion efficiency of LED-pumped laser at $\lambda_{p}=460 \mathrm{~nm}$ is four times greater than the green LED-pumped Nd:YAG laser at $\lambda_{p}=520 \mathrm{~nm}$ [4].

The reasons of laser poor efficiency are inefficient coupling between LEDs and laser rod, and the low pump energy (about 1 Joule), which result a very low optical gain coefficient of the order $g_{o} \approx 0.015 \mathrm{~cm}^{-1}$ and very low stored energy $J_{s t}=g_{o} E_{s}=9.9 \mathrm{~mJ} / \mathrm{cm}^{3}$ in active medium, where $E_{s}=0.66 \mathrm{~J} / \mathrm{cm}^{2}$ is saturation fluence of $\mathrm{Nd}: Y A G$ [12]. Therefore, total stored energy in laser rod is $E_{\text {stored }}=\pi a_{r}^{2} L_{a m} J_{s t}=4.2 \mathrm{~mJ}$ and the laser energy $E_{\text {laser }}=\left(1-R_{o c}\right) E_{\text {stored }}=0.29 \mathrm{~mJ}$ is comparable with $0.32 \mathrm{~mJ}$, the measured energy of laser free-running at $E_{p}=1 J$, Fig. 3 .

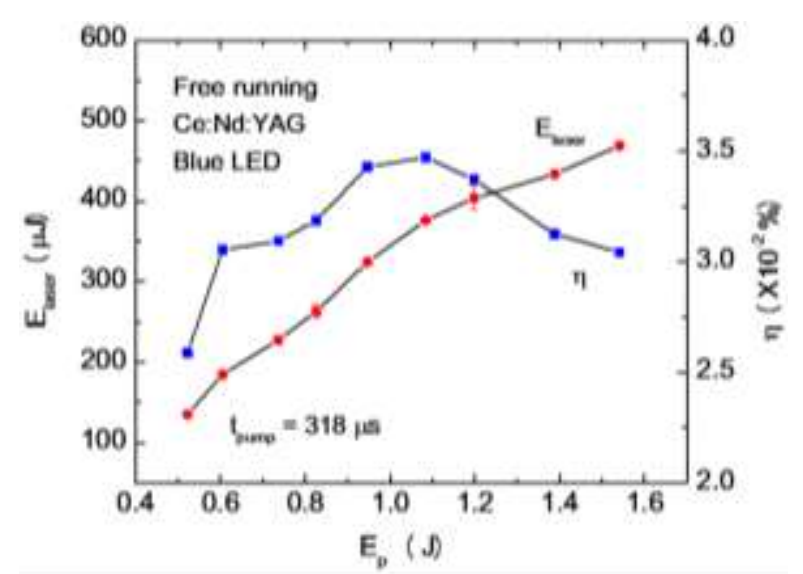

Fig. 3. Energy of the free-running laser spikes and electrical to optical conversion efficiency versus pump energy delivered to the LED bars.

The first spike of laser free-running with 800 ns pulse-width was appeared 67 microseconds after the rising edge of the pumping pulse when 1.2 joules pumping energy delivered to LED bars. Insertion of a blank optical plate into the laser resonator with about 90 percent transmission ceased the laser oscillation. Therefore, the initial transmission of PQS must be greater than 90 percent. In addition, several numerical simulations of section 4 , with different value of $T_{0}$, lead us to use a 
$\mathrm{Cr}^{4+}$ :YAG crystal with $T_{0} \approx 95 \%$ in order to overcome the loss of the resonator and obtain a single Q-switched pulse with pumping rate similar to conditions for laser free-running.

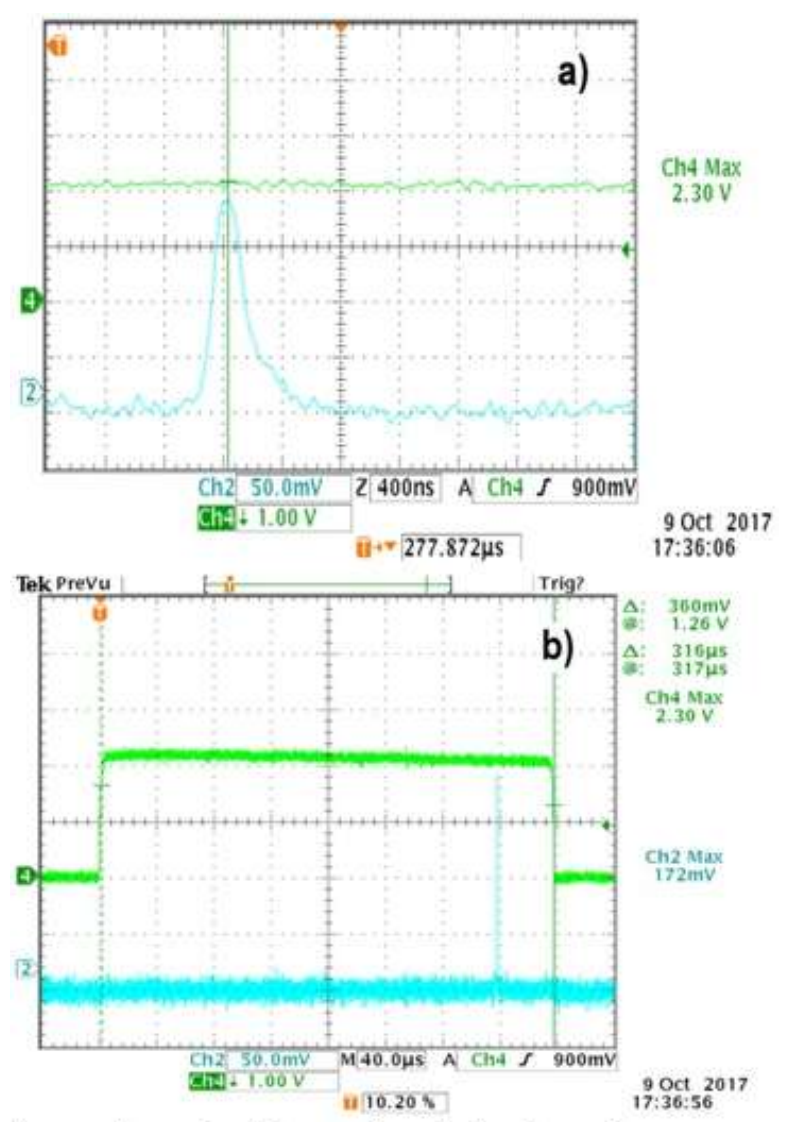

Fig. 4. a) Typical laser time behavior of a $240 \mathrm{~ns}$ single Q-switched pulse slightly above threshold $\left.E_{p}=0.7 \mathrm{~J}, \mathrm{~b}\right)$ the position of the Q-switched pulse is 278 microseconds after the rising edge of the LEDs current pulse.

By using a thin $\mathrm{Cr}^{4+}$ :YAG Q-switch crystal with $T_{0}=96 \pm 1 \%$ where it is placed near the back mirror of the resonator and closely to the laser threshold with $E_{p}=0.7 \mathrm{~J}$, a single Qswitch pulse with $\approx 240 n s$ pulse-width has been observed after 278 microseconds from the leading edge of the LEDs current pulse, Fig. 4.

By increasing the pumping energy, the required time to generate the $\mathrm{Q}$-switched pulse is decreased. Further increasing of $E_{p}$ up to $\approx 1.2 \mathrm{~J}$, nearly two similar laser pulses were generated at 188 and 312 microseconds after the initiation of the pumping pulse, Fig. 5.
Measurements were done by a combination of fast PIN diode and amplifier (less than $5 \mathrm{~ns}$ response time), an attenuator filter with neutral density 1, and a $500 \mathrm{MHz}$ digital oscilloscope.

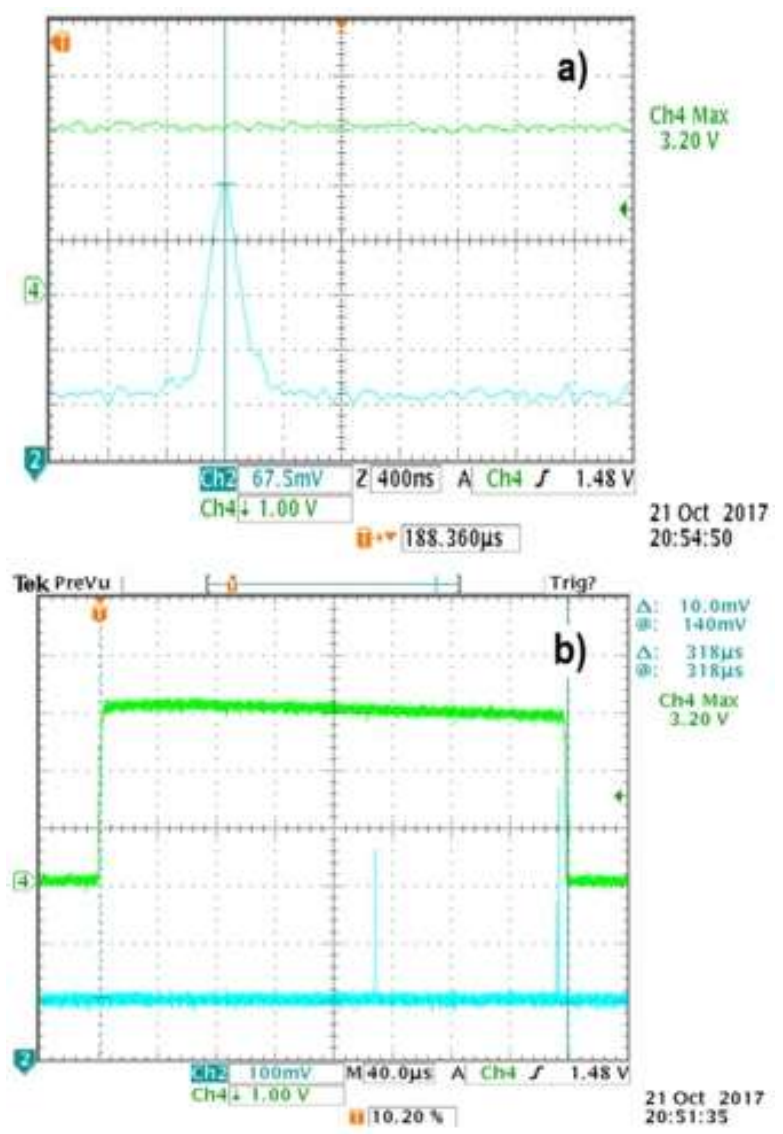

Fig. 5. a) Time behavior of the first Q-switched pulse with $\left.E_{p}=1.2 \mathrm{~J}, \mathrm{~b}\right)$ the position of the first and second Q-switched pulses are respectively 188 and 312 microseconds after the rising edge of LEDs current pulse.

Figure 6 shows the energy and number of the Q-switched laser pulse(s), total LEDs current and conversion efficiency versus pump energy. The maximum energy of a single Q-switched pulse is 23 micro-joules at $E_{p}=0.9 \mathrm{~J}$. The highest value of conversion efficiency $\eta=2.6 \times 10^{-3} \%$ for Q-switching at $E_{p} \approx 1.2 \mathrm{~J}$ is approximately one order of magnitudes lower than the laser free-running. Comparison of the free-running laser spikes with the Qswitched pulses and including the attenuation factor of optical filters, indicates that the laser peak power increased by one order of magnitude [6]. 
The laser mode profile is captured and analyzed with a gentec WinCamD beam diagnostics CCD camera at $25 \mathrm{~cm}$ from the output coupler. The Q-switched laser mode structure is $\mathrm{TEM}_{00}$ for $E_{p}<0.8 \mathrm{~J}$ and otherwise TEM $_{10}$, Fig. 7.
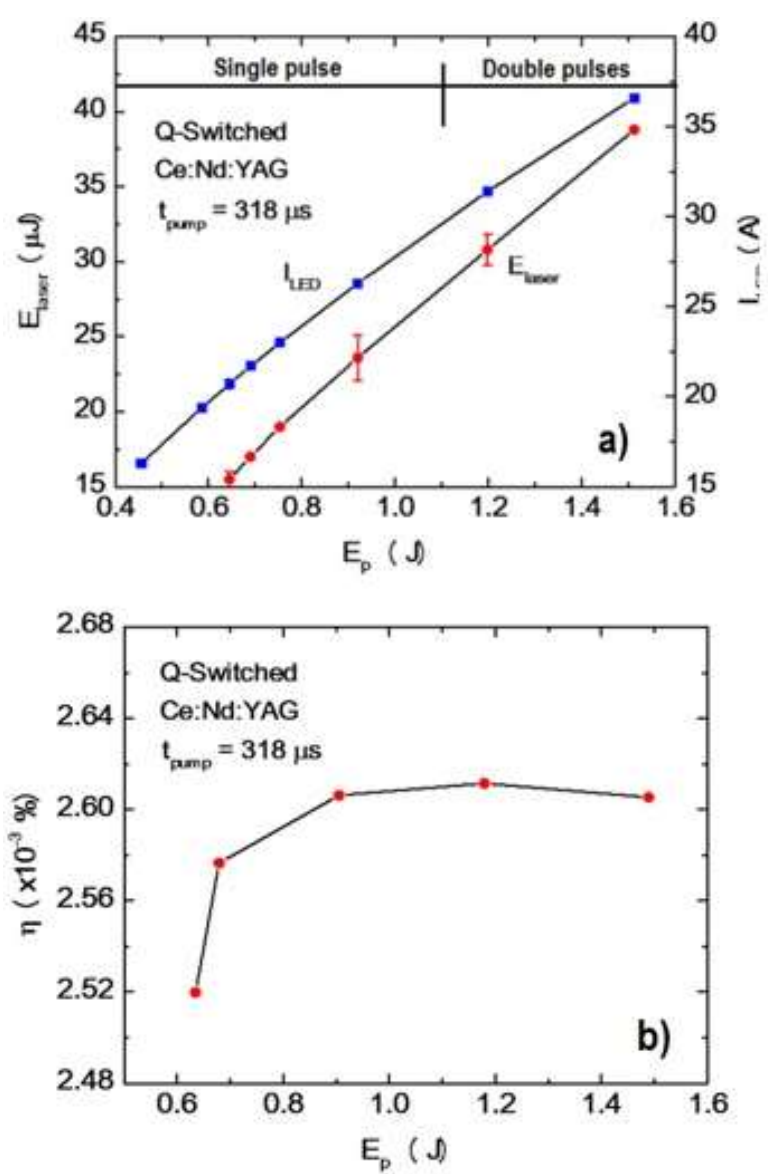

Fig. 6. a) Energy and number of the Q-switched laser pulse(s) versus pump energy and total LEDs current, b) electrical to optical conversion efficiency versus pump energy delivered to the LED bars.

For comparison, the free-running laser mode profiles of Ce:Nd:YAG laser have been captured for different pump energy, Fig. 8. Without the passive Q-switch, the resonator oscillates on high order transverse mode $\mathrm{TEM}_{30}$ when $\mathrm{E}_{\mathrm{p}}$ is less than 0.4 Joules. $\mathrm{TEM}_{31}$ and higher order modes were observed for $E_{p}>0.4 J$. The $\mathrm{TEM}_{00}$ mode has not been achieved for laser free-running.

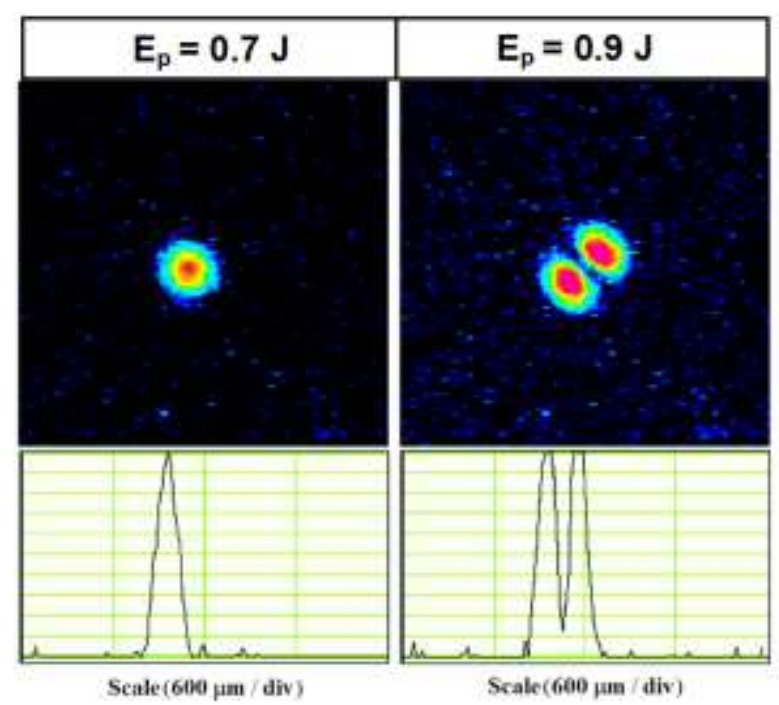

Fig. 7. Mode profiles of the Q-switched LEDpumped Ce:Nd:YAG laser for pump energy equal to $E_{p}=0.7 \mathrm{~J}$ and $E_{p}=0.9 \mathrm{~J}$.

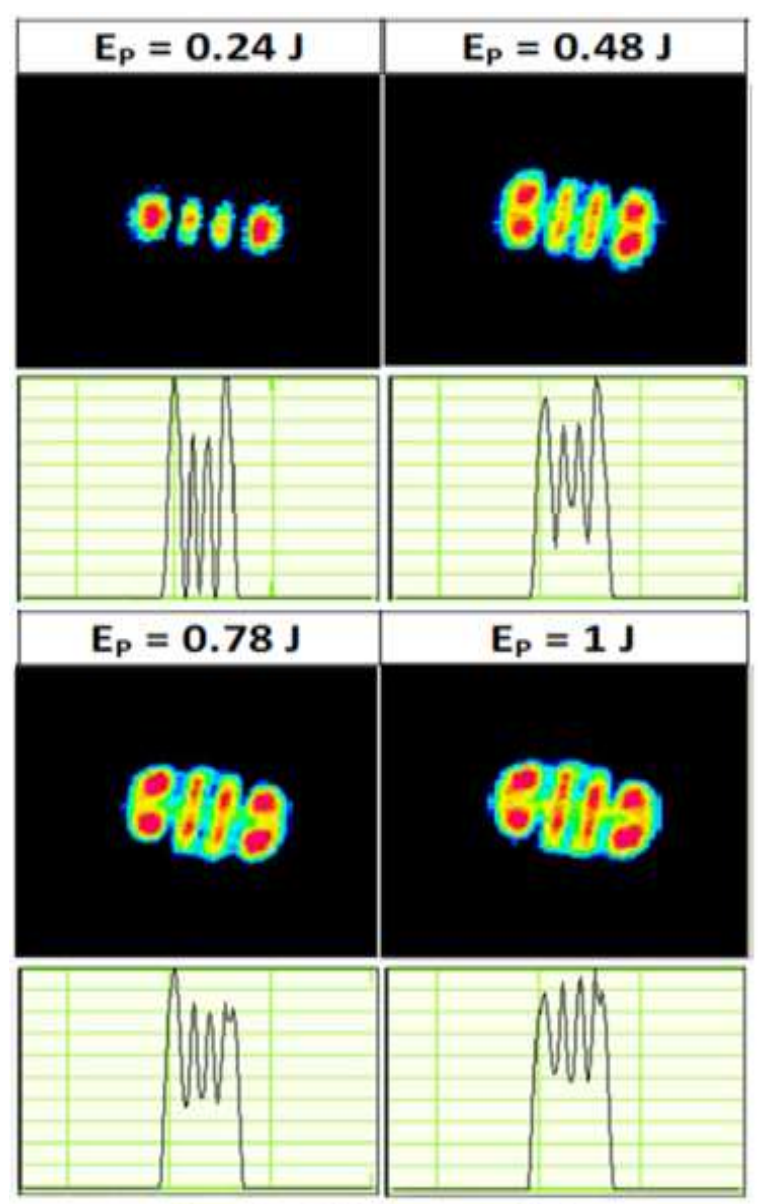

\section{Scale $(800 \mu \mathrm{m} /$ div $)$}

Fig. 8. The Ce:Nd:YAG laser free-running mode profiles along their major axis of symmetry for the different values of pump energy. 


\section{IV.ANALYSIS AND DISCUSSION}

To study the reason of $\mathrm{TEM}_{00}$ mode profile, we have used OptiChamber software [11] to calculate the absorption pumping profile across the transverse section of the laser rod. The software algorithm is based on ray tracing. We have used $5 \times 10^{5}$ emitting rays per LED, 96 radial layers and 96 angular slices for calculations. Moreover, the absorption spectrum of Nd:YAG and Ce:Nd:YAG is used with emission spectral power of LEDs at 592 and $460 \mathrm{~nm}$, respectively. In addition, the precise geometry of pumping configuration is used to obtain reliable results [4].

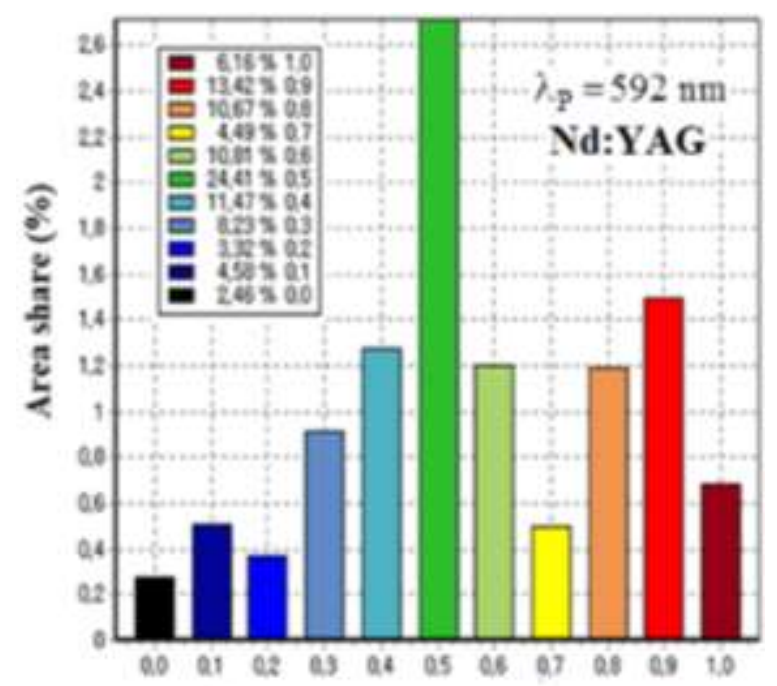

Relative absorbed power level

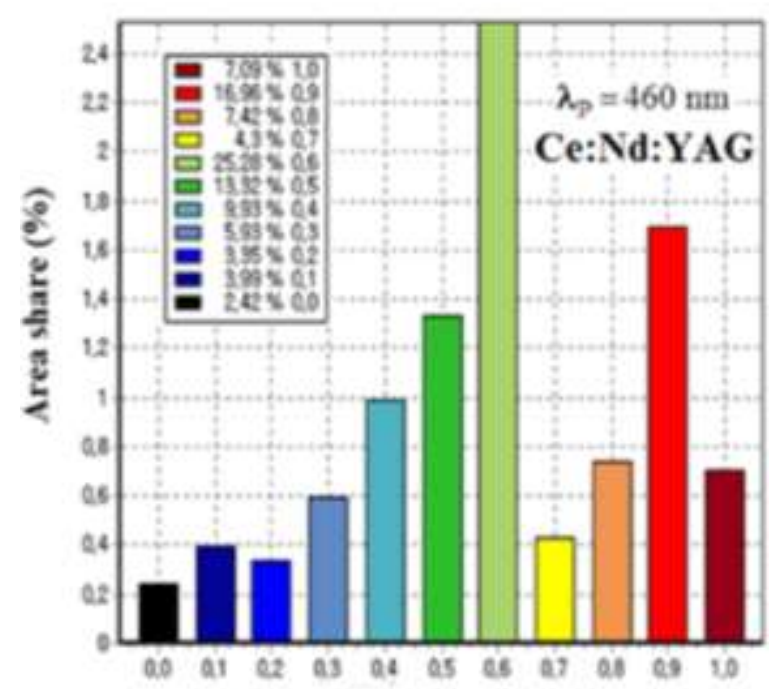

Relative absorbed power level

(a)
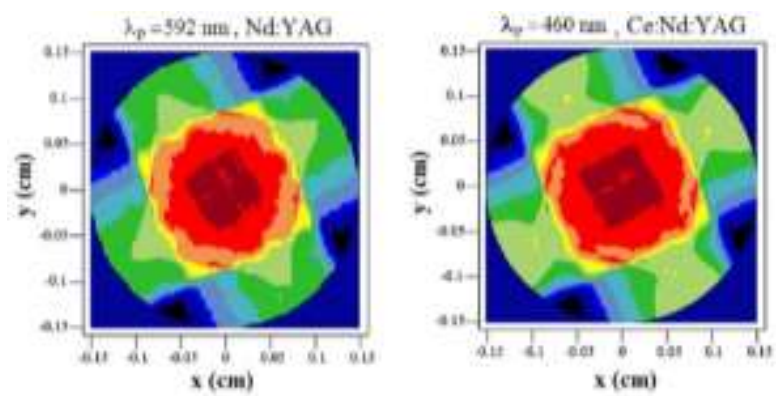

(b)

Fig. 9. The calculated values of: a) absorbed power versus the shared area of laser rod and, b) pumping profile across the cross-section of laser rod for $\mathrm{Nd}: \mathrm{YAG}$ and Ce:Nd:YAG active medium at $\lambda_{p}=592 \mathrm{~nm}$ and $\lambda_{p}=460 \mathrm{~nm}$, respectively.

Figure 9 shows the result of numerical simulations. It is seen from Fig. 9(b) that the central area of laser rod absorbed more pumping power. However, for $1 \mathrm{~W} / \mathrm{cm}$ lineal emitted power of LEDs, pumping power density $\rho_{P}=2.33 \mathrm{~W} / \mathrm{cm}^{3}$ for Ce:Nd:YAG rod, is 6.6 times greater than $\rho_{P}$ of the $\mathrm{Nd}$ :YAG rod under similar lineal emitted power and pumping geometry. Hence, the central part of the Ce:Nd:YAG laser rod has sufficient optical gain to amplify spontaneous emission intensity up to PQS saturation intensity. Therefore, the central area of the laser rod with a radius less than $0.5 \mathrm{~mm}$ operates like as a spatial filter with soft aperture and enforces the laser to oscillate on the lowest order transverse mode.

To study the time behavior of LED-pumped passively Q-switched laser, we have solved the system of rate equations Eq. (2), for the population of energy levels of the active medium $\left(N_{2}, N_{1}\right)$ and the saturable absorber $\left(N_{g s}, N_{e s}\right)$, simultaneously with photon density $\phi$ rate equation by using the finite difference method [10-13].

$$
\begin{aligned}
& \frac{d \phi}{d t}=\left(\frac{L_{r}}{L_{g}}\right) c \sigma \phi\left(N_{2}-\frac{g_{2}}{g_{1}} N_{1}\right) \\
& +\frac{1}{4}\left(\frac{a_{r}}{L_{r}}\right)^{2} \frac{N_{2}}{t_{f}}-\phi\left(\frac{1}{\tau_{c}}+\left(\frac{L_{s a}}{L_{g}}\right) c\left(N_{e s} \sigma_{e s}+N_{g s} \sigma_{g s}\right)\right)
\end{aligned}
$$




$$
\begin{aligned}
\frac{d \phi}{d t}= & \left(\frac{L_{r}}{L_{g}}\right) c \sigma \phi\left(N_{2}-\frac{g_{2}}{g_{1}} N_{1}\right) \\
& -\phi\left(\frac{1}{\tau_{c}}+\left(\frac{L_{s a}}{L_{g}}\right) c\left(N_{e s} \sigma_{e s}+N_{g s} \sigma_{g s}\right)\right) \\
& +\frac{1}{4}\left(\frac{a_{r}}{L_{r}}\right)^{2} \frac{N_{2}}{t_{f}} \\
\frac{d N_{2}}{d t}= & -c \sigma \phi\left(N_{2}-\frac{g_{2}}{g_{1}} N_{1}\right)-\frac{N_{2}}{t_{f}}+W(t) \\
\frac{d N_{1}}{d t}= & c \sigma \phi\left(N_{2}-\frac{g_{2}}{g_{1}} N_{1}\right)-\frac{N_{1}}{\tau_{1}} \\
\frac{d N_{g s}}{d t}= & \frac{N_{e s}-N_{g s}}{\tau_{s}}-c \sigma_{g s} \phi N_{g s} \\
N_{e s}= & N_{o s}-N_{g s}
\end{aligned}
$$

The physical parameters of $\mathrm{Cr}^{4+}: Y A G$ for numerical calculations are taken from Ref. [14-16]. The absorption cross section of the ground state and the excited state for the two levels model of $\mathrm{Cr}^{4+}$ :YAG are respectively $\sigma_{g s}=3.2 \times 10^{-18} \mathrm{~cm}^{2}$ and $\sigma_{e s}=4.5 \times 10^{-19} \mathrm{~cm}^{2}$. The lifetime of PQS excited state is $\tau_{s}=3.6 \times 10^{-6} s$. Total chromium ion density is shown by $N_{o s}=N_{g s}+N_{e s}$. For active medium, the stimulated cross section of $\mathrm{Nd}^{3+}$ in YAG crystal at $1064 \mathrm{~nm}$ is $\sigma=2.8 \times 10^{-19} \mathrm{~cm}^{2}$ and the fluorescence lifetime of upper state ${ }^{4} F_{3 / 2}$, and lower state ${ }^{4} I_{11 / 2}$ lifetime, are $t_{f}=230 \mu \mathrm{s}$ and $\tau_{1}=30 \mathrm{~ns}$, respectively [12]. Moreover, the degeneracy factor of upper and lower states are $g_{2}=4$ and $g_{1}=12$. The velocity of light in the active medium is $c=c_{0} / n_{a m}$, where $c_{0}$ and $n_{a m}=1.82$ are respectively the velocity of light in vacuum and medium index of refraction. The radius and length of laser rod are shown by $a_{r}$ and $L_{r}$, respectively.

The second term at the right-hand side of the photon density rate equation of Eq. (2), $1 / \tau_{\text {eff }}=1 / \tau_{c}+\left(L_{s a} / L_{g}\right) c\left(N_{e s} \sigma_{e s}+N_{g s} \sigma_{g s}\right)$ is the effective cavity photon decay rate. Photon cavity lifetime, $\tau_{c}=t_{r} / \varepsilon$, is related to photon round-trip time $t_{r}=2 L_{\text {opt }} / c_{o}$, and timeindependent loss, $\varepsilon=L_{\text {loss }}-\ln \left(R_{b m} R_{o c}\right)$, where diffraction loss and scattering are included in $L_{\text {loss }} \cdot R_{b m}$ and $R_{o c}$ are the reflectivities of the mirrors.

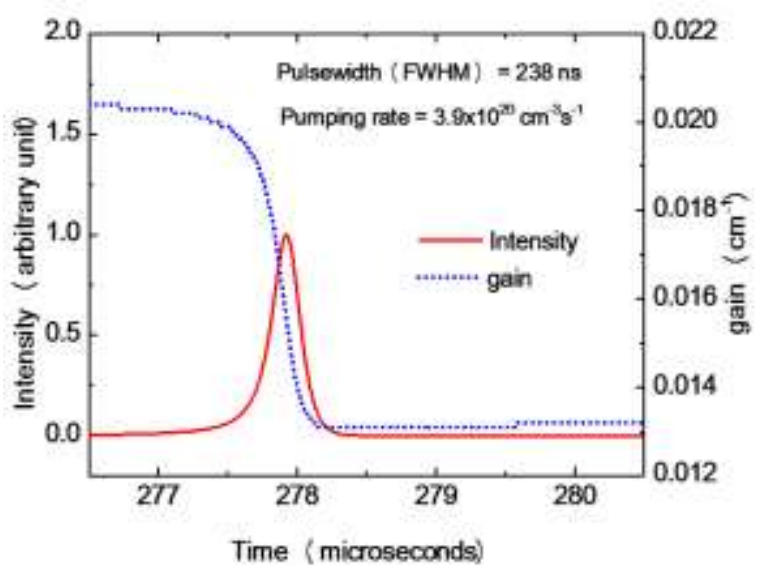

Fig. 10. The calculated laser intensity and optical gain of the Q-switched Ce:Nd:YAG laser with $E_{p}=0.7 \mathrm{~J}$ similar to conditions of Fig. 6 .

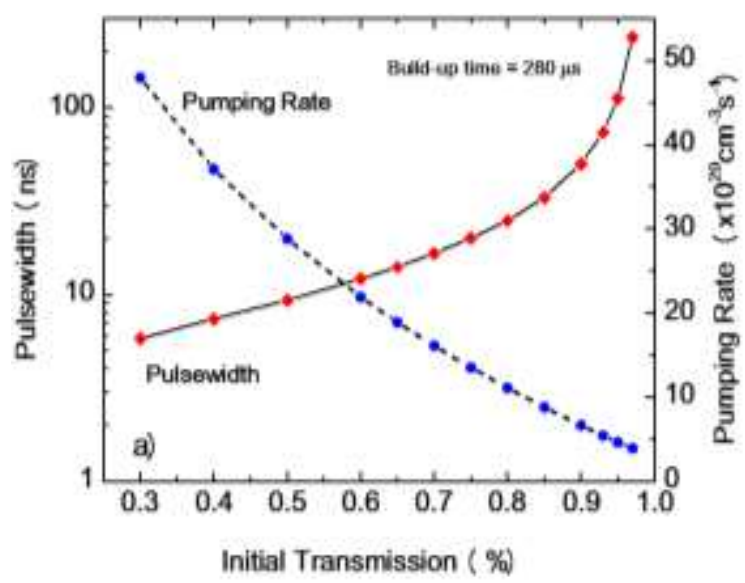

(a)

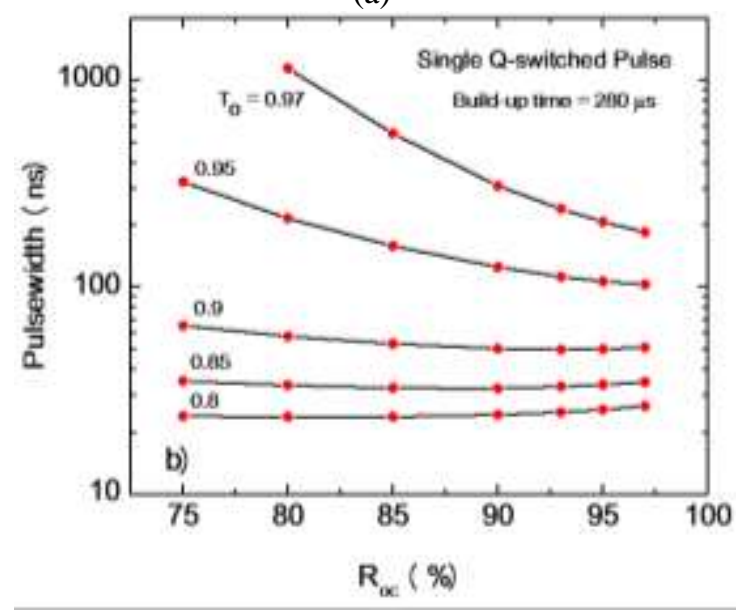

(b) 
Fig. 11. a) Calculated laser pulse-width and required pumping rate versus initial transmission $T_{0}$ of the Cr:YAG passive QS, b) pulse-width versus reflectivity of output coupler for different values of $T_{0}$. For all cases the pulse build up time is $t_{b}=280 \mu s$

The rate of spontaneous emission $\frac{1}{4}\left(a_{r} / L_{r}\right)^{2} N_{2} / t_{f}$ is also included in the photon rate equation [17], and $\mathrm{W}(\mathrm{t})$ is the volumetric pumping rate of the active medium. For actual conditions, the total ion density $N_{o s}=2.25 \times 10^{17} \mathrm{~cm}^{-3}$ is calculated from the Beer-Lambert's law $T_{0}=e^{-\sigma_{e f f} N_{o s} L_{s a}}$ by using $T_{0}=96 \%$ and the value of crystal thickness $L_{s a}=0.66 \mathrm{~mm}$. The effective absorption cross section is $\sigma_{e f f}=\sigma_{g s}-\sigma_{e s}$.

The numerical solution of Eq. (2) for a square pumping pulse $W(t)=W_{o} \mid \begin{array}{ll}1, & 0 \leq t<t_{\text {off }} \\ 0, & t \geq t_{\text {off }}\end{array}$, where $W_{o}=3.9 \times 10^{20} \mathrm{~cm}^{-3} \mathrm{~s}^{-1}, \quad t_{\text {off }}=318 \mu \mathrm{s} \quad$ and internal optical loss coefficient $\alpha_{\text {loss }}=0.0018 \mathrm{~cm}^{-1}$, is shown in Fig. 10. The instant of laser peak power $t_{\text {peak }} \approx 278 \mu \mathrm{s}$, and laser pulse-width $t_{p w}=238 n s$, are very similar to the observed value of Fig. 6. Threshold condition, Eq. (3), give us the approximate value of gain coefficient $g_{T}=0.016 \mathrm{~cm}^{-1}$ that is consistent with the gain value of Fig. 10 at $t=t_{\text {peak }}$.

$g_{T}=\left(2 L_{g} \alpha_{\text {loss }}+2 L_{s a} \sigma_{e f f} N_{o s}-\ln \left(R_{b m} R_{o c}\right)\right) / 2 L_{a m}$

The numerical analysis confirms that the laser pulse-width is highly depended on the initial transmission of passive Q-switch. According to Fig. 11 and Eq. (3), a short Q-switched pulse requires low $T_{0}$ and high gain value. Therefore, in order to reduce laser pulse-width one order of magnitude and obtain a typical 25 ns Q-switched pulse, one requires $T_{0} \approx 0.8$ and the pumping rate must be increased to $W_{0}=11 \times 10^{20} \mathrm{~cm}^{-3} \mathrm{~s}^{-1}$.
By increasing the pump energy, the required time $t_{b}$ to build-up the first Q-switched laser pulse decreases, however, the laser pulsewidth remains nearly constant and has a weak dependency on $R_{o c}$ for $T_{0}<0.9$, Fig. 11(b).

\section{Conclusion}

Frequently, to obtain a single controllable high power laser pulse, the well-known Qswitching method has been used [12]. Experimental findings confirm that the passive Q-switching is a suitable candidate for LEDpumped solid-state lasers to obtain a reliable nanosecond Q-switched laser pulse [18].

We have experimentally studied the pumping of a relatively thick $3 \mathrm{~mm}$ diameter Ce:Nd:YAG laser rod with blue LEDs, and reliable Q-switched pulses at pumping rates $3.5-4.5 \times 10^{20} \mathrm{~cm}^{-3} \mathrm{~s}^{-1}$ with $t_{p w} \approx 240 \mathrm{~ns}$ have been observed. Simulations show that the central high gain area of the laser rod and saturation mechanism of the passive Q-switch behave like as a spatial filter with a soft aperture to enforce laser resonator on a low order transverse modes where results a highquality laser beam.

In addition, the results of numerical solutions of the rate equations that are consistent with the experiments indicate that a single Qswitched laser pulse, as short as 20 ns can be obtained with initial transmission $T_{0} \approx 0.75$ and $W_{0}=13 \times 10^{20} \mathrm{~cm}^{-3} \mathrm{~s}^{-1}$. However, it requires increasing the number of LEDs and using more powerful LED-bars. In addition, the proper imaging of a large number of LED sources is a serious problem where we are trying to solve it with a specially designed optics to couple efficiently and uniformly the pumping radiation to the active medium.

\section{REFERENCES}

[1] A. Barbet, F. Balembois, A. Paul, J.P. Blanchot, A.L. Viotti, J. Sabater, and F. Druon, P. Georges, "Revisiting of LED pumped bulk laser: first demonstration of $\mathrm{Nd}: \mathrm{YVO}_{4}$ LED pumped laser," Opt. Lett. Vol. 39, pp. 6731-6734, 2014. 
[2] B. Villars, E.S. Hill, and C.G. Durfee, "Design and development of a high-power LEDpumped Ce:Nd:YAG laser," Opt. Lett. Vol. 40, pp. 3049-3052, 2015.

[3] K.Y. Huang, C.K. Su, M.W. Lin, Y.C. Chiu, and Y.C. Huang, "Efficient 750-nm LEDpumped Nd:YAG laser," Opt. Express, Vol. 24, pp. 12043-12054, 2016.

[4] M. Tarkashvand, A.H. Farahbod, and S.A. Hashemizadeh, "First demonstration of green and amber LED-pumped Nd:YAG laser," Laser Physics, Vol. 28, pp. 055801 (1-7), 2018.

[5] M. Tarkashvand, A.H. Farahbod, and S.A. Hashemizadeh, "Experimental Study of Passively Q-switched LED-Pumped Solidstate Laser, $24^{\text {th }}$ Iranian Conference on Optics and Photonics and $9^{\text {th }}$ Iranian Conference on Photonics Engineering and Technology," University of Shahrekord, Jan. 30-Feb. 1, 2018.

[6] M. Tarkashvand, A.H. Farahbod, and S.A. Hashemizadeh, "Passively Q-switched LEDpumped Ce:Nd:YAG laser," Iranian Journal of Physics Research, Vol. 18, pp. 477-484, 2018.

[7] C.Y. Cho, C.C. Pu, K.W. Su, and Y. F. Chen, "LED-side-pumped Nd:YAG laser with>20\% optical efficiency and the demonstration of an efficient passively Q-switched LED-pumped solid-state laser," Opt. Lett. Vol. 42, pp. 23942397, 2017.

[8] P. Pichon, A. Barbet, D. Blengino, P. Legavre, T. Gallinelli, F. Druon, J.P. Blanchot, F. Balembois , S. Forget, S. Chénais, and P. Georges, "High-radiance light sources with LED-pumped luminescent concentrators applied to pump Nd:YAG passively Qswitched laser," Opt. Laser Technol. Vol. 96, pp. 7-12, 2017.

[9] C.Y. Cho, C.C. Pu, Y.F. Chen, and K. W. Su, "Energy scale-up and mode-quality enhancement of the LED-pumped Nd:YAG Qswitched laser achieving a millijoule green pulse," Opt. Lett. Vol. 44, pp. 3202-3205, 2019.

[10] Y. Li, S. Zhou, H. Lin, X. Hou, and W. Li, "Intense $1064 \mathrm{~nm}$ emission by the efficient energy transfer from $\mathrm{Ce}^{3+}$ to $\mathrm{Nd}^{3+}$ in $\mathrm{Ce} / \mathrm{Nd}$ four YAG transparent ceramics," Opt. Mater, Vol. 32, pp. 1223-1226, 2010.
[11] OptiChamber 2014 Software (version 2.1) for Modeling Laser-Pumping Trains (C) Centre "TOM" (C) 2000-2014, Centre "Technology of Optical Materials," 36/1 Babushkina str. St.Petersburg 193171, Russia.

[12] W. Koechner, Solid-State Laser Engineering, $6^{\text {th }}$ Ed. Springer, 2006.

[13] J.J. Degnan, "Optimization of Passively QSwitched Lasers," IEEE J. Quantum Electron. Vol. 31, pp. 1890-1901, 1995.

[14] X. Zhang, S. Zhao, Q. Wang, Q. Zhang, L. Sun, and S. Zhang, "Optimization of $\mathrm{Cr}^{4+}$ doped saturable-absorber Q-switched lasers," IEEE J. Quantum Electron. Vol. 33, pp. 22862294, 1997.

[15] J. Dong, "Numerical modeling of CW-pumped repetitively passively Q-switched Yb:YAG lasers with Cr:YAG as saturable absorber," Opt. Commun. Vol. 226, pp. 337-344, 2003.

[16]Y. Kalisky, A. Ben-Amar Baranga, Y. Shimony, and M.R. Kokta, " $\mathrm{Cr}^{4+}$ doped garnets: novel laser materials and non-linear saturable absorbers," Opt. Mater. Vol. 8, pp.129-134, 1997.

[17]G. Ghani Moghadam and A.H. Farahbod, "General formula for calculation of amplified spontaneous emission intensity," Opt. Quant. Electron. Vol. 48, pp. 227 (2-6), 2016.

[18] J. Chen and J.N. Chen, "Five Simultaneously Q-Switch Mode-Locked Passive Laser Modulators," Opt. Rev. Vol. 13, pp. 427-435, 2006.

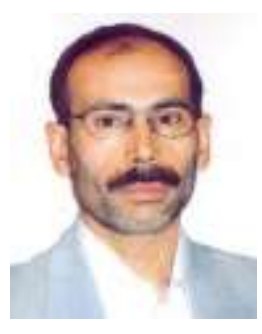

Mostafa Tarkashvand joined to department of optics and laser (Tehran-Iran), at the beginning of 1980s, where he made several kinds of nitrogen and dye lasers. Then, he started to fabricate and characterized AlGaAs based semiconductor lasers by use of liquid phase epitaxy reactor. 
By joining to plasma and nuclear fusion research school, at the beginning of 2000s, he worked on solid-state lasers, especially on flashlamp pumped $\mathrm{Nd}$ :YAG and $\mathrm{Nd}$ :Glass lasers.

At 2015, he decided to pump solid-state lasers by visible light emitting diodes, which for several years he intended to do it. At 2016 he succeed to do this job as his $\mathrm{PhD}$ thesis subject, and consequently received his $\mathrm{PhD}$ degree in physics from Payame Noor University, Tehran, Iran, at 2020. He is currently working on development of LED pumped solid-state lasers to make them commercially available devices.

Amir Hossein Farahbod, photograph and biography not available at the time of publication.

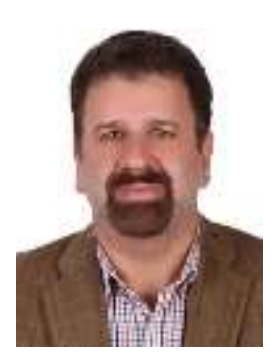

Seyed Ali Hashemizadeh is Associate Professor of Condensed Matter Physics at Payame Noor University. His subjects of interests are Semiconductor Devices, LEDs, Semiconductor Lasers, Solar Cells and Sensors. 